\title{
Stage III Extrahepatic Bile Duct Cancer
}

National Cancer Institute

\section{Source}

National Cancer Institute. Stage III Extrahepatic Bile Duct Cancer. NCI Thesaurus. Code C5771.

Stage III includes: T4, Any N, M0. T4: Tumor invades any of the following main portal vein or its branches bilaterally, common hepatic artery, or other adjacent structures, such as the colon, stomach, duodenum, or abdominal wall. N0: No regional lymph node metastasis. N1: Regional lymph node metastasis. M0: Distant metastasis. (AJCC 6th ed.) 2003 